\title{
Resonant Plasmonic and Vibrational Coupling in a Tailored Nanoantenna for Infrared Detection
}

\author{
Frank Neubrech and Annemarie Pucci* \\ Kirchhoff Institute for Physics, University of Heidelberg, Im Neuenheimer Feld 227, 69120 Heidelberg, Germany \\ Thomas Walter Cornelius and Shafqat Karim \\ Gesellschaft für Schwerionenforschung, Planckstrasse 1, 64291 Darmstadt, Germany
}

Aitzol García-Etxarri and Javier Aizpurua

Donostia International Physics Center and Centro de Física de Materiales CSIC-UPV/EHU, Paseo Manuel de Lardizabal 4, Donostia-San Sebastián 20018, Spain

(Received 12 February 2008; published 7 October 2008)

\begin{abstract}
A novel resonant mechanism involving the interference of a broadband plasmon with the narrowband vibration from molecules is presented. With the use of this concept, we demonstrate experimentally the enormous enhancement of the vibrational signals from less than one attomol of molecules on individual gold nanowires, tailored to act as plasmonic nanoantennas in the infrared. By detuning the resonance via a change in the antenna length, a Fano-type behavior of the spectral signal is observed, which is clearly supported by full electrodynamical calculations. This resonant mechanism can be a new paradigm for sensitive infrared identification of molecular groups.
\end{abstract}

PACS numbers: 78.67.Lt, 33.70.-w, 73.20.Mf, 78.30.-j

Vibrational spectroscopy of molecules is of general importance in natural sciences, medicine, and technology. Direct infrared (IR) observation of molecular vibrations from a reduced number of molecules is a current challenge in all these fields. The respective sensitivity can be increased by several orders of magnitude with the use of surface-enhanced scattering techniques such as surfaceenhanced Raman scattering (SERS) [1-4] and surfaceenhanced IR absorption (SEIRA) [5-12]. The enhancement in these techniques essentially depends on the capacity of the environment to enhance the local electromagnetic (EM) fields. In SERS, the EM field enhancement is effective for both the incoming and the scattered radiation, and usually the local field enhancement profits from plasmon cavity modes with resonant wavelengths in the visible [13]. For direct excitation of vibrational modes, as is the case in SEIRA, the EM field enhancement requires resonant excitation in the IR, because the absorption scales with the intensity of the local field created at the molecules position. Until now, no more than 3 orders of magnitude of direct vibration signal enhancement have been achieved in typical SEIRA experiments [5-12] where a metal-particle ensemble is used as substrate. Its spectral response is strongly broadened by the mutual particle interaction and, additionally, by inhomogeneous particle geometries. The measured SEIRA signal is collected from a macroscopic area (typically a few $\mathrm{mm}^{2}$ ) and represents an average over a variety of different sites with different maximum near-field enhancement factors at spectral positions far away from the frequencies of the molecular vibrations. In this Letter we experimentally show that the effect of the resonant coupling of an individ- ual plasmonic IR nanoantenna with the vibrational excitation produces a different type of resonant SEIRA with unprecedented signal enhancement of 5 orders of magnitude, which means attomol sensitivity. The enhancing effect occurs only when the resonant interaction between both excitations (antenna and molecular vibration) is achieved, as proven by our calculations. We adopt a novel approach to generate half-wave dipolelike antenna resonances in the IR by means of $\mu \mathrm{m}$-long gold nanowires (NW) [14]. Enhancing effects of such gold nanoantennas are a matter of increasing interest in nanooptics [15-17]. To achieve a completely resonant situation, the length $L$ of the nanoantenna is designed to hold a plasmonic resonance exactly matching the spectral position of the vibrational fingerprints. Because of the finite negative value of the dielectric response of gold in the IR, the field penetrates a few $10 \mathrm{~nm}$ into the metal, shifting the strong plasmonic antenna excitations to longer resonance wavelength $\lambda_{r}$ compared to the ideal case; i.e., antenna resonances in the $\mu \mathrm{m}$ range of the spectrum appear for slightly shorter $L$ than the ideal half-wave dipole antenna length $[14,16,18,19]$. With help of exact EM calculations that correctly predict the spectral resonance position, we are able to design the geometrical characteristics of the nanoantenna to obtain the resonance at the required IR wavelength. Figure 1 depicts the calculated enhancement of the near-field EM intensity around such a NW (length $L=$ $1.31 \mu \mathrm{m})$ at its fundamental dipolelike resonance $\left(\lambda_{r} \approx\right.$ 2.6L). The IR plasmon field of the antenna resonance produces an exceptionally large intensity enhancement at the tip ends [17]. As we will show, this localized EM field, resonantly coupled to the molecular vibrations, provides 


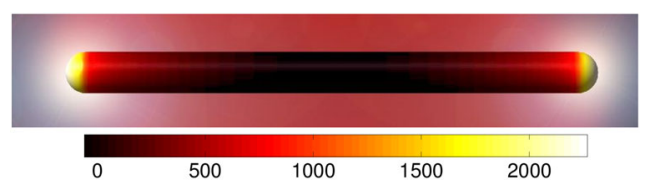

FIG. 1 (color online). Calculation of the near-field intensity $1 \mathrm{~nm}$ above the surface of a gold NW (in vacuum, $L=1.31 \mu \mathrm{m}$, diameter $D=100 \mathrm{~nm}$ ) at the fundamental antenna resonance $\left(\lambda_{r}=3.41 \mu \mathrm{m}\right)$. The color scale (shading) indicates the magnitude of the enhancement.

sufficient enhancement to be used as a sensitive spectroscopic tool for straightforward identification of molecular species.

We perform IR microspectroscopy (probe-area diameter $8 \mu \mathrm{m}$ ) of individual gold NWs (diameter $D=100 \mathrm{~nm}$, prepared by electrochemical deposition in etched ion-track membranes, supported by a transparent substrate) at the IR beam line of the synchrotron light source ANKA (Angströmsource Karlsruhe) [14,20]. The NWs consist of only a few crystalline grains and their tip shape results from a relative smooth grain boundary [21]. The relative IR transmittance spectrum (at normal incidence, divided by the spectrum of the bare substrate) of an individual metal NW prepared with a length of a few microns (see Fig. 2) shows a broad plasmonic feature for parallel (II) polarization of the IR radiation, associated with the fundamental antenna mode (see Fig. 2). This resonance, also known as the longitudinal fundamental plasmonic mode, is suppressed for perpendicular polarization $(\perp)$ of the incoming IR radiation (red line in Fig. 2) and the respective transmittance change to the bare substrate is not detected. For ideal antennas on a substrate, the wavelength of the fundamental antenna resonance of a NW with $L$ and $D \ll L$ can be roughly approximated with the relation $\lambda_{r} / n_{\text {eff }}=$ $2 L$ using an effective refractive index $n_{\text {eff }}$ that accounts for

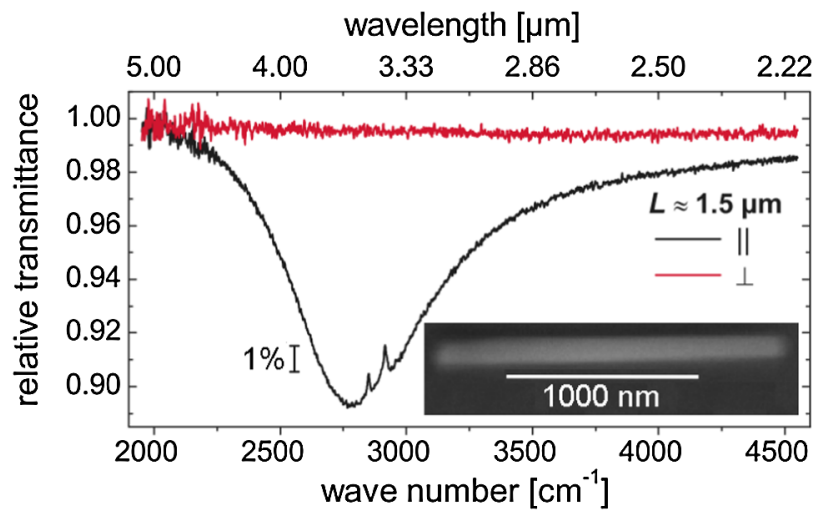

FIG. 2 (color online). Relative IR transmittance in the spectral region of the fundamental resonance of a gold NW with one ODT monolayer for parallel ( $\|$ ) and perpendicular polarization $(\perp)$. $\mathrm{A} \mathrm{CaF}_{2}$ substrate is used. The broadband plasmonic resonance is observed around $\lambda_{r} \approx 3.6 \mu \mathrm{m}(\|)$. Inset: Scanning electron micrograph of a gold NW with similar dimensions as used in this study. the substrate polarizability [14]. The exact wavelength of the plasmon resonance in vacuum is furthermore shifted due to (i) the nonperfect conductive nature of gold at IR frequencies, (ii) the actual aspect ratio, and (iii) the nonnegligible skin depth compared to $D$. Thus, in our case, an about $1.5 \mu \mathrm{m}$ long $\mathrm{NW}$ on $\mathrm{CaF}_{2}\left(n_{\text {eff }}=1.22\right)$ shows the resonant peak at $\lambda_{r} \approx 3590 \mathrm{~nm}\left(2800 \mathrm{~cm}^{-1}\right)$, in agreement with full EM calculations [14,19]. The extinction cross section of the individual wire obtained from the broad feature of reduced intensity in the transmittance curve in Fig. 2 turned out to be enhanced with respect to the geometrical cross section, which clearly indicates the activation of an intense near field in the vicinity of the wire (simple shadowing does not give a measurable transmittance change). The experimental cross section ratio (Fig. 3) is a far-field result and represents an average extinction value over a large interval of distances at the wire surface. The intensity of the spatially dependent near-field enhancement that is mainly localized at a few $\mathrm{nm}$ distance from the antenna-tip ends can reach up to 4 orders of magnitude, as theoretically determined and indicated in Fig. 1. We used this localized plasmonic IR field to enhance the spectral signature of molecular vibrations. One layer of octadecanthiol [ODT, $\mathrm{CH}_{3}\left(\mathrm{CH}_{2}\right)_{16} \mathrm{CH}_{2} \mathrm{SH}$ ] was used as model ad-
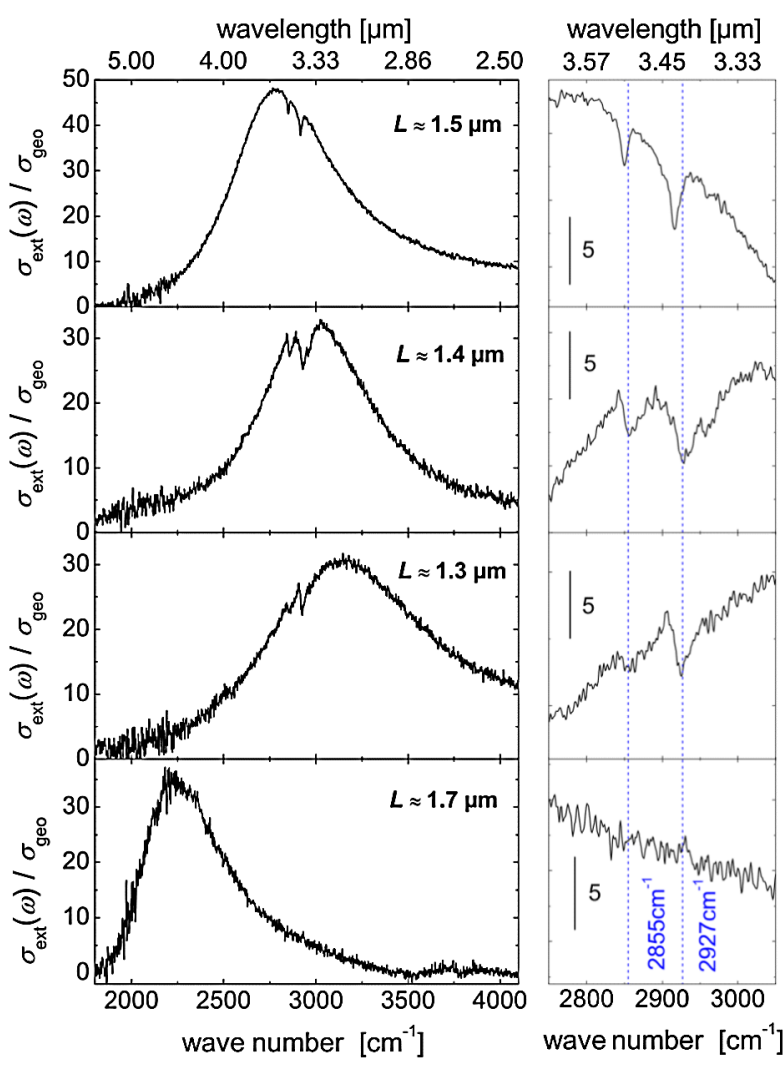

FIG. 3 (color online). Experimental extinction cross sections $\sigma_{\text {ext }}$ (normalized to the geometrical cross section $\sigma_{\text {geo }}$ ) for four ODT-coated wires with different $L$ as indicated and $D=$ $100 \mathrm{~nm}$ on $\mathrm{CaF}_{2}$. The noise level slightly varied depending on the incoming IR intensity. To the right, the zoom to the vibrational signals. Dotted lines: ODT vibration frequencies [6]. 
sorbate. ODT molecules were adsorbed on individual gold NWs and the IR transmittance was measured with the IRmicrospectroscopy setup. The long-chained ODT molecules form a self-assembled monolayer (SAM) with the molecular axis slightly inclined with respect to the surface normal [22]. The layer has a thickness of $2.4 \mathrm{~nm}$ [23]. ODT exhibits several IR active vibration modes in the $3 \mu \mathrm{m}$ wavelength range. For our investigation, the strongest absorption modes of the $\mathrm{CH}_{2}$ group [antisymmetric $\left(2916-2936 \mathrm{~cm}^{-1}\right)$ and symmetric $\left(2850-2863 \mathrm{~cm}^{-1}\right)$ stretching vibrations [24]] are considered. The resonant coupling between the fundamental NW resonance and the ODT bands was studied for NWs with different $L$, i.e., resonance frequencies showing different spectral shifts with respect to the $\mathrm{CH}_{2}$ frequencies. Figure 3 shows relative extinction cross sections (in the far field) as derived from IR transmittance [14] for the different individual NWs covered with a SAM of ODT. Obviously, the good match of the antenna resonance to the $\mathrm{C}-\mathrm{H}$ stretching vibration lines is crucial for the appearance of the vibrational signal. The vibrational signal slightly decays as the resonance is slowly detuned (for $L=1.3 \mu \mathrm{m}$, and $L=$ $1.5 \mu \mathrm{m})$, and cannot be detected for the strongly detuned case (Fig. 3, curve for an antenna with $L=1.7 \mu \mathrm{m}$ ). For electric field perpendicular to the wire no vibration lines could be observed. From the experimental observations, we conclude that the vibrations observed in Figs. 2 and 3 are the result of the interaction of the nanoantenna that enhances the signal above the noise level. The signal size (as difference between maximum and minimum transmittance at the vibration feature) reaches values of about $1 \%$ for $L=1.5 \mu \mathrm{m}$, see Fig. 2, and $1.6 \%$ for the best match. By comparison to IR reflection absorption spectra (IRRAS) from a SAM of ODT on a smooth gold surface [6] the vibration signal size for the best match turns out to be about 330000 times bigger than it would be for the same amount of molecules directly on the substrate (without antenna) [20]. It is important to note that the signal comes mainly from the area of the tip ends of the NW, where the strongest field enhancement is produced (see Fig. 1), corresponding to about 150000 molecules (less than one attomol). Interestingly, the line shape of the vibration signal clearly differs from a typical Lorentzian absorption band. Asymmetric vibrational line shapes are already known, e.g., from SEIRA on metal-island films [25,26]. In this study, the special line shape of the adsorbate signal results from the interaction between the EM field from the resonance of an individual nanoantenna and the spectrally much narrower field associated with the vibrational dipoles of the molecules. The spectral shift for different nanoantennas produces differences in the coupling that transform the spectral line shape accordingly. This situation is a clear analogy to the quantum mechanical interaction between a discrete state and a continuum of states producing Fano profiles [27]. Depending whether the antenna resonance and the vibrations are exactly matching or not, the Fano-type profile goes from a dip to an asymmetric line shape, respectively. As shown in Fig. 3, the overall extinction cross section produced by the antenna for an exactly resonant situation $(L=1.4 \mu \mathrm{m})$ shows several dips, i.e., zones of smaller extinction at the vibration frequencies, which can be interpreted as a result of an antiphase interaction of both kinds of excitation (vibrational and plasmonic). The dips in the spectrum correspond to a resonant situation where the interaction between the broadband and narrowband resonances is maximum, therefore producing a Fano-like antiresonance (dip), whereas a more asymmetric Fano-like profile is obtained as we depart from resonance (weaker interaction) $[27,28]$. The observed vibration line shape can be theoretically reproduced when the full EM interaction of the antenna and the ODT layer is considered, as shown in Fig. 4. The calculations were performed by means of a boundary element method (BEM) [29] to solve Maxwell's equations exactly for the scattering of a gold NW covered with a molecular layer. We use IR bulk dielectric functions to characterize the response of gold [30], and a Lorentz oscillator model with a dielectric background to characterize the vibrational response of the ODT. In this case the oscillator parameters were obtained from a fit to experimental IRRAS [31]. The

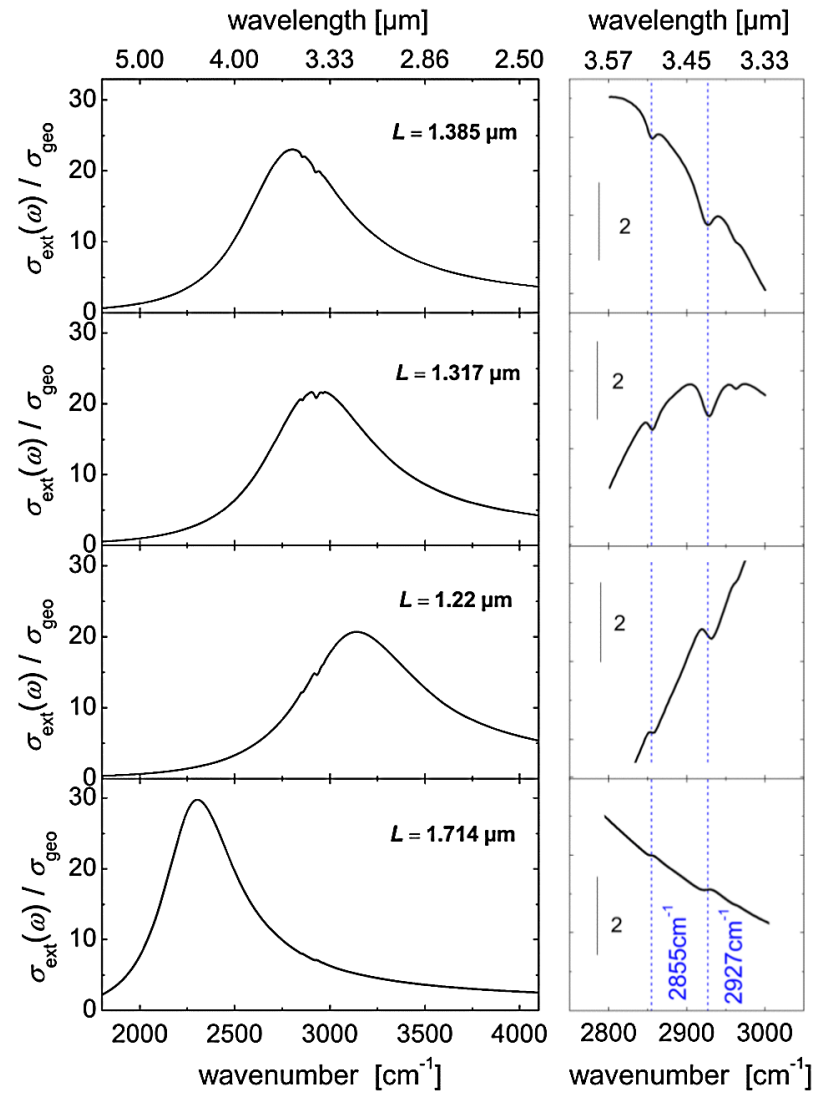

FIG. 4 (color online). Calculated resonance curves for gold NWs with $D=100 \mathrm{~nm}$, covered with $100 \mathrm{~nm}$ ODT layer, for $L$ as indicated, and surrounded by vacuum. The BEM calculations are performed for polarization along the long wire axis. To the right, zoom into the vibrational bands. Dotted lines mark ODT vibrational frequencies. 
oscillator-strength parameters of such fit turned out to be sensitive to the assumptions made for the dielectric background. The results for the extinction cross section of an ODT-covered nanoantenna using oscillator strengths parameters from an IRRAS fit with the background value $\varepsilon_{b}=1$ are shown in Fig. 4. A vibrational contrast comparable to the resonance experiment is reached only for a relative thick ODT layer. A similar contrast in theory and experiment would be obtained for a single monolayer with ODT oscillator parameters that were calculated from IRRAS assuming a higher $\varepsilon_{b}$, which points to remarkable screening of molecules at metal surfaces. The anisotropy of the molecular monolayer does not significantly modify the contrast obtained with the use of an isotropic dielectric function. Also the detailed tip shape (semispherical or more flat) does not give significant effect on the IR resonance and on the Fano-type effect. Chemical effects as dynamic charge transfer $[32,33$ ] can be excluded to have any relevance for the measured response, because the $\mathrm{CH}_{2}$ groups producing the signal are not directly bound to the gold. The results from the model calculations corroborate the experimental findings: For an antenna resonantly matching its plasmonic broadband resonance with vibrational narrowbands of molecules $(L=1.317 \mu \mathrm{m}$ in Fig. 4), maximum contrast is obtained with strong dips in the spectrum. These antiresonance features appear exactly at the vibrations frequencies. As the length of the antennas is increased or reduced, the broadband plasmonic resonance is spectrally shifted to the red or to the blue with respect to the vibrations, producing a change in the phase interaction (modification of the coupling strength in terms of Fano interaction) and progressively decreasing vibrational signals with clearly asymmetric profiles. These line shapes correspond to Fano-like profiles of intermediate interaction. The asymmetry of the line shapes is reversed when the broadband plasmonic resonance is located to the right or to the left of the vibrations. For a completely detuned antenna $(L=1.714 \mu \mathrm{m})$, the asymmetric vibrational contrast is almost vanished, in agreement with the experiments. In a similar detuning fashion, calculations for perpendicular polarization do not provide measurable vibration signals. The generation of the resonant situation is therefore crucial to obtain the enormous enhancement of the signal and maximum contrast.

In conclusion, IR vibration signals with enormous enhancement of 5 orders of magnitude can be achieved with resonant interaction of vibration dipoles with a broadband IR plasmonic antenna resonance of a gold NW, which can be interpreted as classical analogy to the antiresonances produced in the Fano effect. This enhancement mechanism enables a new powerful technique for surface-enhanced IR scattering with general importance for a variety of fields. By exploiting the resonant enhancement in the vibrational spectra, as shown in this Letter, it will be possible to detect and to study extremely small quantities of molecules, thus lowering the detection limit of direct IR vibration spectroscopy considerably.
F. N. and T. C. gratefully acknowledge technical support by M. Süpfle, helpful discussions with B. Gasharova and Y.-L. Mathis (ANKA). Acknowledgements for financial support are for F.N. and A.P. from the DFG (DFG Pu 193/9), for S. K. from the Higher Education Commission (HEC), Pakistan, and for J. A. from the ETORTEK project of the Government of the Basque Country.

*pucci@kip.uni-heidelberg.de

[1] M. Moskovits, Rev. Mod. Phys. 57, 783 (1985).

[2] A. Otto, J. Raman Spectrosc. 22, 743 (1991).

[3] S. M. Nie and S. R. Emory, Science 275, 1102 (1997).

[4] K. Kneipp et al., Phys. Rev. Lett. 78, 1667 (1997).

[5] H. Wang, J. Kundu, and N. J. Halas, Angew. Chem. 46, 9040 (2007).

[6] D. Enders and A. Pucci, Appl. Phys. Lett. 88, 184104 (2006).

[7] R. Aroca, Surface-Enhanced Vibrational Spectroscopy (John Wiley \& Sons, Ltd., New York, 2006).

[8] R. Aroca, Appl. Spectrosc. 58, 324A (2004).

[9] N. Goutev and M. Futamata, Appl. Spectrosc. 57, 506 (2003).

[10] T. R. Jensen et al., Appl. Spectrosc. 54, 371 (2000).

[11] M. Osawa and M. Ikeda, J. Phys. Chem. 95, 9914 (1991).

[12] M. Osawa, in Near-Field Optics and Surface Plasmon Polaritons, Topics in Applied Physics Vol. 81, edited by S. Kawata (Springer, Berlin/Heidelberg, 2001), pp. 163-187.

[13] H. Xu et al., Phys. Rev. Lett. 83, 4357 (1999).

[14] N. Neubrech et al., Appl. Phys. Lett. 89, 253104 (2006).

[15] N. Yu et al., Appl. Phys. Lett. 91, 173113 (2007).

[16] P. Mühlschlegel et al., Science 308, 1607 (2005).

[17] T. H. Taminiau et al., Nano Lett. 7, 28 (2007).

[18] J. Aizpurua et al., Phys. Rev. B 71, 235420 (2005).

[19] G. W. Bryant et al., Nano Lett. 8, 631 (2008).

[20] See EPAPS Document No. E-PRLTAO-101-036842 for supplementary material containing details on measurements, samples, and signal enhancement. For more information on EPAPS, see http://www.aip.org/pubservs/ epaps.html.

[21] S. Karim et al., Appl. Phys. A 84, 403 (2006).

[22] F. Schreiber, Prog. Surf. Sci. 65, 151 (2000).

[23] E. K. Seo et al., Chem. Mater. 16, 1878 (2004).

[24] D. Lin-Vien, N. W. Colthup, W. G. Fateley, and J.G. Grasselli, The Handbook of Infrared and Raman Frequencies of Organic Molecules (John Wiley \& Sons, Ltd., New York, 1991).

[25] A. E. Bjerke et al., Anal. Chem. 71, 1967 (1999).

[26] A. Priebe et al., J. Chem. Phys. 119, 4887 (2003).

[27] U. Fano, Phys. Rev. 124, 1866 (1961).

[28] J. Aizpurua et al., Opt. Express 16, 1529 (2008).

[29] F. J. García de Abajo and A. Howie, Phys. Rev. Lett. 80, 5180 (1998).

[30] E. D. Palik, Handbook of Optical Constants of Solids (Academic, New York, 1985).

[31] SCOUT software package, M. Theis Hard- and Software, Aachen.

[32] A. Otto, J. Raman Spectrosc. 36, 497 (2005).

[33] A. Priebe et al., J. Phys. Chem. B 110, 1673 (2006). 